\title{
Evolution of Catalyst Coated Atomised Magnesium Spheres - an alternative thermal storage medium for concentrated solar power applications
}

\author{
Priyen C. Mistry, David M. Grant, Alastair D. Stuart, Kandavel Manickam, \\ Gavin S. Walker \\ Advanced Materials Research Group, Faculty of Engineering, University of Nottingham, \\ University Park, Nottingham, NG7 2RD, UK
}

\begin{abstract}
Elevated temperature cycling studies were performed on two commercial gas atomised Mg spherical powders (average diameter of $26 \mu \mathrm{m}$ and $30 \mu \mathrm{m}$ ) with magnetron sputtered catalysts (chromium, iron, vanadium and stainless steel) applied to their surfaces. At $350{ }^{\circ} \mathrm{C}$, the presence of a catalyst promotes faster reaction kinetics with improving capacity until approaching stabilisation by the $90^{\text {th }}$ cycle, e.g. the normalised capacity of V_Mg30 was found to rise from $45.5 \%$ to $65.5 \%$. Following determination of activation energies (from Kissinger plots) and microstructural analysis of the post cycled structures a mechanism was proposed for the differing evolutions of the uncoated and coated Mg powders based upon a complex process in which particle sintering competes with particle fragmentation. Catalyst effectiveness varied with temperature, having a negligible impact on hydrogen storage characteristics of the atomised $\mathrm{Mg}$ powders following 50 cycles at $400{ }^{\circ} \mathrm{C}$ and this was mainly associated with the lack of multivalency in the catalysts.

Keywords: Thermal storage, Catalysis, Atomised, Hydrogen, Magnesium, Magnetron sputtering
\end{abstract}

\section{Introduction}

In order to reduce global $\mathrm{CO}_{2}$ emissions, the International Energy Agency predicts approximately a tenth of global electricity generation will be from renewable concentrated solar power (CSP) by 2050 [1]. However, widespread deployment of CSP at an industrial level is impeded by the mismatch between 
solar generation and peak electrical demand, effectively driving generation costs far above those of conventional power plants $\left(\$ 0.06 \mathrm{kWh}^{-1}\right)[2]$. Thermal storage offers a solution to this predicament by retaining appropriate amounts of heat for sustained periods enabling CSP plants to deliver dispatchable power.

Existing CSP thermal energy stores, such as molten salts and phase change materials, that exploit sensible and latent heat material properties respectively, have been demonstrated to be viable but exhibit intrinsically low energy densities such as $60 \% \mathrm{NaNO}_{3}-40 \% \mathrm{KNO}_{3}$ which obtains a volumetric energy density of approximately $290 \mathrm{MJ} \mathrm{m}^{-3}[3,4]$. Thermochemical storage can reach energy densities at least one order of magnitude higher. The highly reversible $\mathrm{Mg}-\mathrm{MgH}_{2}$ metal-hydride system is particularly promising, obtaining an enthalpy of reaction of $74.5 \mathrm{~kJ} \mathrm{~mol}^{-1} \mathrm{H}_{2}$ that translates to a volumetric energy density of approximately $4080 \mathrm{MJ} \mathrm{m}^{-3}$ of $\mathrm{MgH}_{2}$. Nevertheless, magnesium is hindered by slow activation and poor kinetics of (de)hydrogenation, even when approaching temperatures ideal for CSP applications (in the region $\left.400{ }^{\circ} \mathrm{C}\right)[5]$.

The fabrication of nanocrystalline structures via severe plastic deformation (SPD) techniques, most commonly by ball milling, is a well known approach for accelerating the dissociation and diffusion of hydrogen in Mg. Various studies have proven a relationship exists between reaction kinetics and the particle - and grain size of $\mathrm{MgH}_{2}$. Zaluska et al. [6] reported the hydrogenation rate of micron-sized $\mathrm{Mg}$ powder at $300{ }^{\circ} \mathrm{C}$ over a $2 \mathrm{~h}$ period can be increased from $<0.25$ wt. $\%$ to over 6 wt. $\%$, purely through the application of milling to generate $\mathrm{Mg}$ powder with an approximate grain size of $30 \mathrm{~nm}$. These improvements were explained by increased specific surface area and the introduction of defects which provided nucleation sites for hydride formation and new diffusion channels $[7,8]$. As a result, the optimum nanocrystalline structure should combine in the right proportion: a high surface-to-volume ratio to encourage hydrogen dissociation at the surface, alongside a large number of grain boundaries to increase the diffusion pathway density thus assisting hydrogen atom permeation into the particles' bulk [9].

While nanocrystalline $\mathrm{Mg}$ structures have yielded some promising results, there remains uncertainty surrounding the scalability of the SPD technologies involved. In the event of the emergence of a hydrogen economy relying heavily on solid-state storage, economical production of large quantities of metal hydride material becomes a critical factor [10]; hence alternate production techniques must be explored. Gas powder atomisation is a proven method 
capable of powder fabrication in tonnage quantities and overcomes the low efficiencies and complicated procedures encountered with other rapid solidification options. General gas atomisation is tailored to generate consistently spherical powders with particle sizes typically varying from 10-500 $\mu \mathrm{m}$, of which the 10-60 $\mu \mathrm{m}$ range accounts for approximately $50 \%$ of total yield [11].

Since being used to investigate the formation and decomposition of magnesium hydride in the 1980's [12], to our knowledge, there has been no published research into the direct feasibility of atomised $\mathrm{Mg}$ powder as a thermal storage medium. The utilization of atomised $\mathrm{Mg}$ powder may have a multitude of advantages: firstly spherical powders tend to flow more consistently than coarse, irregular powders thus simplifying the filling of a storage vessel, and secondly micron-sized powders help to minimise health and safety concerns associated with potentially pyrophoric and explosive $\mathrm{Mg}$ nano-particulates. Furthermore, the direct use of $\mathrm{Mg}$ opposed to $\mathrm{MgH}_{2}$ as a starting material and the elimination of any SPD technique underlines potential reductions in fabrication costs.

Despite this, the smooth and spherical traits inherent to atomised $\mathrm{Mg}$ powder contradict the high surface area prerequisite for fast activation and kinetics $[13,14]$. In addition, the hydrogen storage properties of $\mathrm{Mg}$ powder has been observed to degrade during successive cycling $[15,16]$. The reasoning was two-fold: a compaction of the powder with continuous expansion and contraction of the particles, thus inhibiting hydrogen flow into the bed and sintering of the compacted particles leading to increased diffusion path length for hydrogen.

Catalysts in the form of transition metal or metal oxides dispersed evenly within a Mg matrix represent another means of enhancing kinetic behaviour $[17,18,19]$, as well as deterring degradation during cycling [20, 21], yet their effects on micron-sized $\mathrm{Mg}$ powder remain to be tested. If identical properties are attainable, it could provide a more viable and scalable approach for fabricating catalysed thermal storage media compared to SPD processed $\mathrm{Mg}$. Therefore, in this work we investigate the influences of magnetron sputtered catalysts applied to the surface of commercially available atomised Mg powder during successive cycling; the aim of which was to fabricate hydrogen storage materials via an alternative processing pathway that possess (de)hydrogenation characteristics equal to or surpassing their nanocrystalline equivalents. 


\section{Experimental}

\subsection{Preparation}

Two particle sizes of commercial grade atomised magnesium (purity >99\%) were purchased from Advanced Powder Technologies and Société pour la Fabrication du Magnésium. Particle size analysis using a Malvern Mastersizer 2000 determined the mean particle sizes $\left(\mathrm{D}_{50}\right)$ to be $30 \mu \mathrm{m}$ and $26 \mu \mathrm{m}$, and from here forth will be referred to as Mg30 and Mg26 respectively. The two as received (AR) Mg powders were passivated prior to being coated with a selection of catalyst candidates as presented in Table 1. All coating work was outsourced to Teer Coatings Ltd, Miba Coating Group, who utilised a modified UDP450 closed field unbalanced magnetron sputter ion plating system based on an argon plasma. The system was capable of deposition on micron-sized powders initially in batches up to $50 \mathrm{~g}$, although a fixed substrate mass of $7 \mathrm{~g}$ was maintained for each coating.

\subsection{Cycling}

All samples were subjected to isothermal cycling using a commercial Sieverts type apparatus (Hy-Energy LLC. PCTPro-2000) with the pressure measured by a capacitance manometer with an accuracy of $1 \%$ of reading. Successive hydrogenations and dehydrogenations were performed using starting pressures of 40 bar and 0.1 bar of hydrogen respectively for a period of 30 min per half cycle. Using these conditions, several sets of cycling experiments were carried out at $350{ }^{\circ} \mathrm{C}$ and $400{ }^{\circ} \mathrm{C}$. In each case uncoated control samples (Mg30 \& Mg26) were tested as base references.

\begin{tabular}{ccccc}
\hline Sample code & Target & $\begin{array}{c}\text { Purity [\%] } \\
\text { (Source) }\end{array}$ & Deposition Conditions & EDX Conc. [wt.\%] \\
\hline V_Mg30 & Vanadium & $\begin{array}{c}99.5 \\
\text { (Kurt J Lesker) }\end{array}$ & $150 \mathrm{~W} \times 4.5 \mathrm{~h}$ & $0.93 \pm 0.12$ \\
SS_Mg30 & Stainless Steel & $\begin{array}{c}\text { Unknown } \\
99.9\end{array}$ & $100 \mathrm{~W} \times 1 \mathrm{~h}+150 \mathrm{~W} \times 4 \mathrm{~h}$ & $3.14 \pm 0.66$ \\
Fe_Mg30 & Iron & $\begin{array}{c}\text { (Kurt J Lesker) } \\
99.95\end{array}$ & $100 \mathrm{~W} \times 2 \mathrm{~h}$ & $0.82 \pm 0.28$ \\
Cr_Mg30 & Chromium & $\begin{array}{c}\text { (PI-KEM Ltd) } \\
\text { 99.95 }\end{array}$ & $150 \mathrm{~W} \times 4.5 \mathrm{~h}$ & $2.32 \pm 0.40$ \\
Cr_Mg26 & Chromium & (PI-KEM Ltd) & $150 \mathrm{~W} \times 3.33 \mathrm{~h}$ & $1.58 \pm 0.18$ \\
\hline
\end{tabular}

Table 1: Magnetron sputtering deposition conditions applied to commercially available gas atomised Mg powders. 
During the first set of experiments at $350{ }^{\circ} \mathrm{C}$, samples $(2.50 \pm 0.05 \mathrm{~g})$ underwent a soaking process prior to cycling, which entailed exposure to 40 bar of hydrogen at $350{ }^{\circ} \mathrm{C}$ until fully saturated or approaching saturation for the uncoated control samples. Following this, the samples were cycled 90 times and subjected to a final extended hydrogenation cycle for $14 \mathrm{~h}$.

A second set of experiments were undertaken at a higher temperature of $400{ }^{\circ} \mathrm{C}$. These samples $(1.00 \pm 0.05 \mathrm{~g})$ were soaked and then cycled 50 times before undergoing a final hydrogenation cycle for $14 \mathrm{~h}$ and shall be referred to as 'soak \& cycled'. A repeat of the $400{ }^{\circ} \mathrm{C}$ experiment was carried out again but with the exclusion of the soaking process and these samples shall be referred to as 'cycled'.

\subsection{Characterisation}

A scanning electron microscope (SEM, Philips XL 30 ESEM-FEG) equipped with energy dispersive analysis of X-rays (EDX) was employed to study structural morphology, in addition to catalyst concentration and distribution. Each solid sample produced by cycling at $350{ }^{\circ} \mathrm{C}$ (which had taken on the cylindrical shape of the sample holder) was cut into half using a scalpel. One of the remaining segments of material was mounted on to an $\mathrm{Al}$ stub using conductive carbon cement and carbon coated for cross-sectional SEM analysis at what was the midpoint of the sample.

The dehydrogenation reaction of the hydride samples produced during the $350{ }^{\circ} \mathrm{C}$ experiments were analysed by differential scanning calorimetry (DSC, Netzsch 204 HP Pheonix). Heating rates of 3, 5, 10 and $20^{\circ} \mathrm{C} \mathrm{min}{ }^{-1}$ from room temperature up to $525{ }^{\circ} \mathrm{C}$ under 1 bar of flowing Ar at a rate of $100 \mathrm{ml} \mathrm{min}{ }^{-1}$ were used to construct Kissinger plots to determine the activation energy of dehydrogenation for each sample. Thermogravimetric analysis (TGA, Netzsch 209 F1 Iris) of the hydride samples were also carried out using a heating rate of $10{ }^{\circ} \mathrm{C} \mathrm{min}-1$ from room temperature up to $550{ }^{\circ} \mathrm{C}$, under 1 bar of flowing Ar at a rate of $100 \mathrm{ml} \mathrm{min}^{-1}$. Powder X-ray diffraction (XRD; Bruker D8 Advance, $\mathrm{Cu} \mathrm{K}_{\alpha}$ source: $\lambda=1.5418 \AA$ ) measurements were conducted on the post cycled dehydrogenated samples for phase identification $(40 \mathrm{kV}, 35 \mathrm{~mA}, 0.6 \mathrm{~mm}$ slit size). The X-ray intensity was measured over a $2 \theta$ range from $30^{\circ}$ to $80^{\circ}$ with a step size of $0.05^{\circ}$ and a dwell time of $4 \mathrm{~s}$. The powdered sample was dispersed onto a crystal silicon wafer in an argon glove box $\left(\mathrm{O}_{2}<0.1 \mathrm{ppm}, \mathrm{H}_{2} \mathrm{O}<0.1 \mathrm{ppm}\right)$ and covered with a thin amorphous tape to minimize oxidation during analysis. 


\section{Results}

\subsection{Hydrogen Storage Characteristics}

Figure 1 displays the initial hydrogenation of samples at $350{ }^{\circ} \mathrm{C}$ versus Mg30 at $400{ }^{\circ} \mathrm{C}$ also undergoing the soak process. Prior to hydrogenation both Mg26 and Mg30 at $350{ }^{\circ} \mathrm{C}$ undergo an incubation period lasting a minimum of $2.5 \mathrm{~h}$. Subsequent to this, Mg26 approaches complete saturation after $44 \mathrm{~h}$, however $\mathrm{Mg} 30$ is yet to reach $90 \%$ of normalized capacity in the same duration. In contrast, hydrogenation at $400{ }^{\circ} \mathrm{C}$ resulted in a very rapid activation (taking ca. a quarter of the time). The influence of catalytic coatings to promote activation is unmistakable with a significant reduction in the incubation periods and halving of hydrogenation times. In fact, the incubation period appears to be completely eliminated for SS_Mg30, in turn causing it to obtain the highest initial rate of hydrogenation $\left(31.5 \% \mathrm{~h}^{-1}\right.$ during the initial $90 \%$ of uptake) and accomplish saturation more rapidly than even $\mathrm{Mg} 30$ at $400{ }^{\circ} \mathrm{C}\left(17.9 \% \mathrm{~h}^{-1}\right.$ during the initial $90 \%$ of uptake).

The hydrogen storage capacity results (example shown in Figure 2(a)) have been converted into normalized capacity ranging from ca. 20-80\% depending on cycling variables such as catalyst concentration and temperature. The kinetics of reaction is typically defined by the time to achieve $90 \%$ of the available reaction value $[16,22]$. Figure 2(b) illustrates the typical

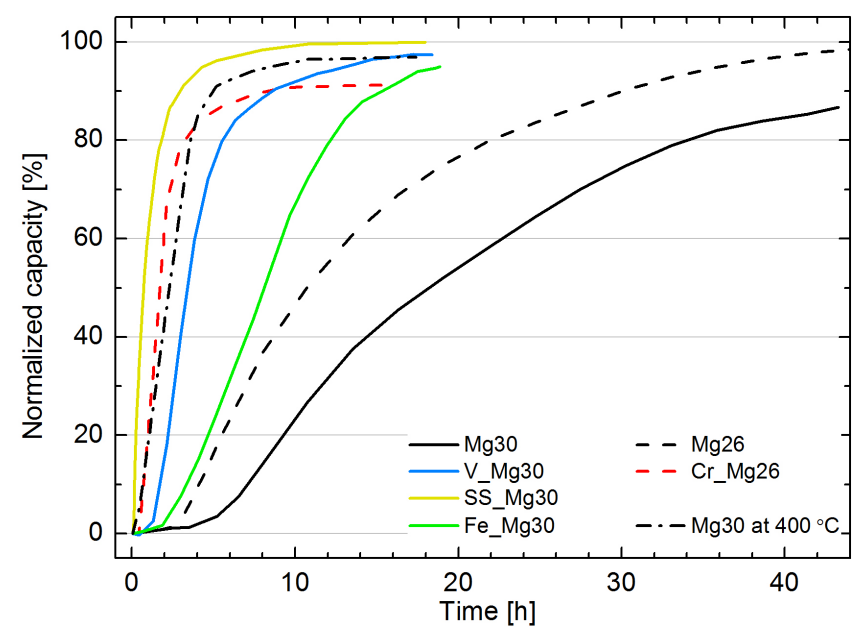

Figure 1: Normalized capacity versus time for each sample during the soaking process for activation at $350{ }^{\circ} \mathrm{C}$ and a starting pressure 40 bar. The hydrogenation curve of Mg30 at $400{ }^{\circ} \mathrm{C}$ has been included as a reference. 

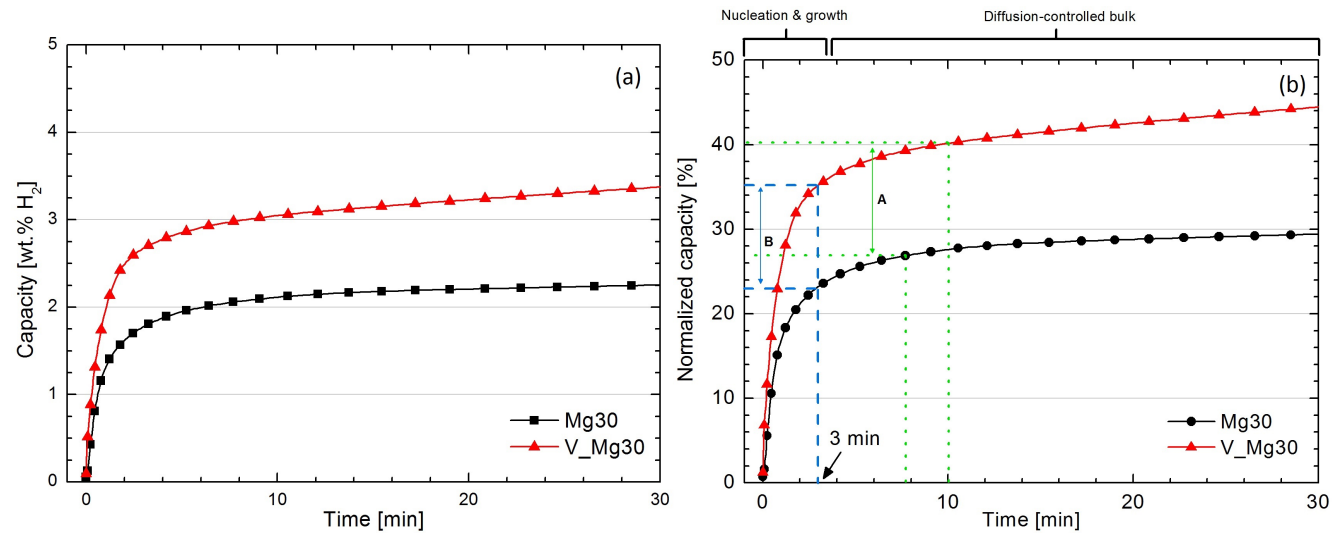

Figure 2: (a) Hydrogen storage capacity and (b) normalized capacity versus time for soak \& cycled Mg30 and V_Mg30 during the $10^{\text {th }}$ hydrogenation cycle at $350{ }^{\circ} \mathrm{C}$. Dotted lines represent $90 \%$ of the available reaction and dashed lines represent 3 min capacities.

hydrogenation kinetics for both the uncoated (Mg30) and vanadium coated (V_Mg30) samples during the $10^{\text {th }}$ cycle at $350{ }^{\circ} \mathrm{C}$, where normalized capacity is displayed on the y-axis. The 3 min marker helps to highlight the distinct change in the hydrogenation rate determining step between magnesium and hydrogen from the initial nucleation stage to diffusion-controlled growth taking place entirely by interface migration of hydrogen from the particle surface [14] (see Figure 2(b)). Although only a small uptake disparity exists between $90 \%$ of the available reactions (marked A) and the 3 min capacities of both

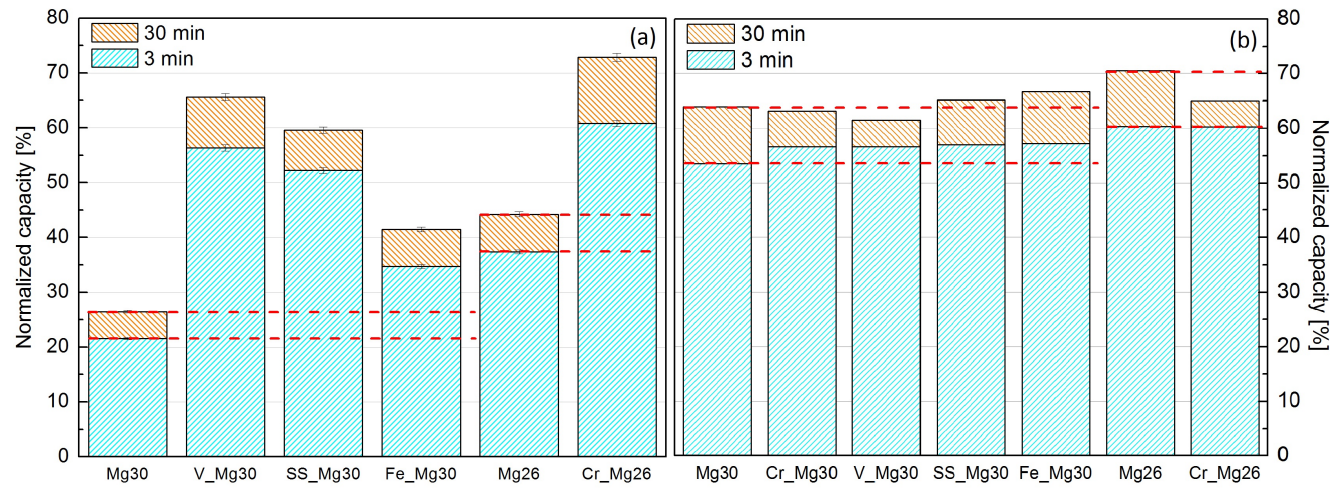

Figure 3: Normalized capacity after 3 and 30 min for each soak \& cycled sample at $350{ }^{\circ} \mathrm{C}$ during the $90^{t h}$ hydrogenation cycle and (b) $400{ }^{\circ} \mathrm{C}$ during the $50^{t h}$ hydrogenation cycle. 
samples (marked B), the bearing on time is more significant. This is because for these atomised powders $90 \%$ of the available reaction appears to be well into the slower 'diffusion-controlled bulk' stage of hydrogenation. Based on these results, it was judged more appropriate to define the hydrogenation kinetics after 3 min and capacities during cycling after 30 minutes.

The hydrogenation characteristics of the samples during their $90^{\text {th }}$ cycle at $350{ }^{\circ} \mathrm{C}$ have been compared in Figure 3(a). As anticipated, cycling of the finer Mg26 led to an improvement in both kinetics and capacity by approximately 17\%. Interestingly, V_Mg30 and Cr_Mg26 exhibit higher total capacities then when they were cycled at $400{ }^{\circ} \mathrm{C}$ (Figure $3(\mathrm{~b})$ ) reaching $65.5 \%$ and $73 \%$ respectively. Furthermore, the capacity of $\mathrm{Cr} \_\mathrm{Mg} 26$ was found to even exceed the best sample at $400{ }^{\circ} \mathrm{C}, \mathrm{Mg} 26$, despite being cycled at $50{ }^{\circ} \mathrm{C}$ lower.

Figure 4 shows the $1^{\text {st }}, 2^{\text {nd }}, 10^{\text {th }}, 30^{t h}, 45^{\text {th }}$ and final $90^{\text {th }}$ cycles for both Mg_30 and V_Mg30 as a function of time at $350{ }^{\circ} \mathrm{C}$. In the $1^{\text {st }}$ cycle, while both samples exhibit a crossover in the hydrogenation rate determining step during the initial 3-5 minutes, the total capacity of V_Mg30 exceeded Mg30 by approximately 18.5\%. Contrasting trends are observed in successive cycles demonstrating the fundamentally different hydrogenation behaviour experienced by the two samples. In the case of Mg30, the capacity rose marginally in the $2^{\text {nd }}$ cycle before eventually dropping to slightly below that measured during the $1^{\text {st }}$ cycle. A much more drastic change is seen for V_Mg30, with the normalized capacity reducing from $45.5 \%$ to $42.5 \%$ in the $2^{\text {nd }}$ cycle after which uptake continued to markedly improve, approaching
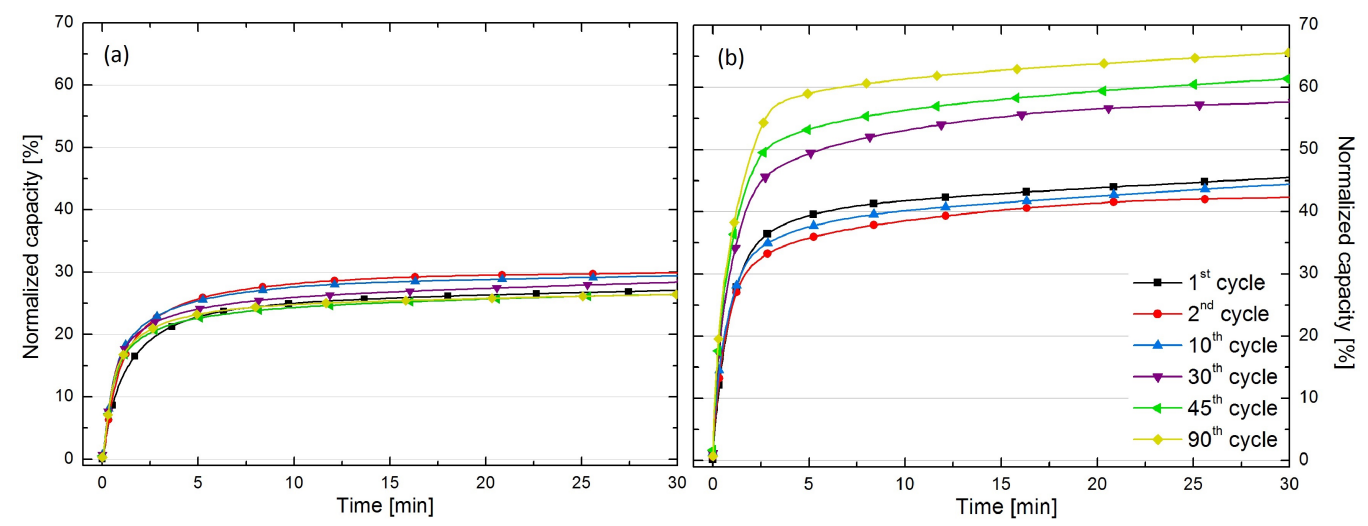

Figure 4: Comparing the normalized capacity versus time during selected cycles for (a) Mg30 and (b) V_Mg30 when cycled at $350{ }^{\circ} \mathrm{C}$. 


\begin{tabular}{cccc}
\hline Sample name & $\begin{array}{c}\text { PCTpro extended normalized } \\
\text { capacity at } 350{ }^{\circ} \mathrm{C}[\%]\end{array}$ & $\begin{array}{c}\text { TGA normalized } \\
\text { capacity }[\%]\end{array}$ & $\begin{array}{c}\mathrm{E}_{a} \\
{\left[\mathrm{~kJ} \mathrm{~mol}^{-1}\right]}\end{array}$ \\
\hline Mg30 & $72.9 \pm 0.7$ & $72.2 \pm 1.3$ & $169 \pm 16$ \\
V_Mg30 & $77.9 \pm 0.8$ & $73.9 \pm 1.3$ & $124 \pm 8$ \\
SS_Mg30 & $74.7 \pm 0.7$ & $73.1 \pm 1.4$ & $118 \pm 22$ \\
Fe_Mg30 & $76.3 \pm 0.8$ & $76.0 \pm 1.3$ & $137 \pm 20$ \\
Mg26 & $83.0 \pm 0.8$ & $81.7 \pm 1.3$ & $169 \pm 9$ \\
Cr_Mg26 & $84.6 \pm 0.8$ & $82.6 \pm 1.3$ & $112 \pm 13$ \\
\hline
\end{tabular}

Table 2: Table comparing the samples capacities measured volumetrically (14 h extended hydrogenation following 90 cycles on PCTpro) and gravimetrically (TGA dehydrogenation taken after the $14 \mathrm{~h}$ hydrogenation measurements). It also shows the activation energies of the samples dehydrogenation reaction derived from Kissinger plots.

$65.5 \%$ by the $90^{\text {th }}$ cycle.

Table 2 illustrates that the total capacity of all of the coated samples exceed that of their uncoated versions when cycled at $350{ }^{\circ} \mathrm{C}$ and after $14 \mathrm{~h}$ Cr_Mg26 continues to obtain the highest capacity at approximately $84.6 \%$. These final hydrogen storage capacities were validated by gravimetric measurements during heating of the samples to $550{ }^{\circ} \mathrm{C}$ in the TGA, the results of which are also shown in Table 2. Using the peak temperatures obtained from the DSC endotherms of the $350{ }^{\circ} \mathrm{C}$ hydride samples (Supplementary Information Figure S1) Kissinger plots were then constructed (Figure S2). The resulting activation energy of dehydrogenation for each sample is also shown in Table 2.

Figure 5 demonstrates the impact of the soaking process prior to cycling at $400{ }^{\circ} \mathrm{C}$ by examining hydrogenation kinetics and total capacity as a function of cycle number for Mg30 and V_Mg30. By the $5^{\text {th }}$ cycle, both the hydrogenation kinetics and total capacity of the cycled Mg30 match those of the soak \& cycled Mg30, after which they follow the same trend with increasing cycle number. Almost identical behaviour can be seen for catalyst coated $\mathrm{Mg}$ samples such as V_Mg30.

The hydrogenation characteristics of the soak \& cycled samples during their $50^{\text {th }}$ cycle at $400{ }^{\circ} \mathrm{C}$ have been compared in Figure 3(b). As observed in Figure 3(a), cycling of the finer Mg26 led to faster kinetics and a higher capacity relative to Mg30 by $6.5 \%$. The kinetics of the coated samples were found to improve by only $3-4 \%$ and in the case of $\mathrm{Cr} \_\mathrm{Mg} 26$ no kinetic improvement was exhibited with respect to Mg26. No clear trend is evident between the capacities of the uncoated and coated $\mathrm{Mg}$ samples after 30 

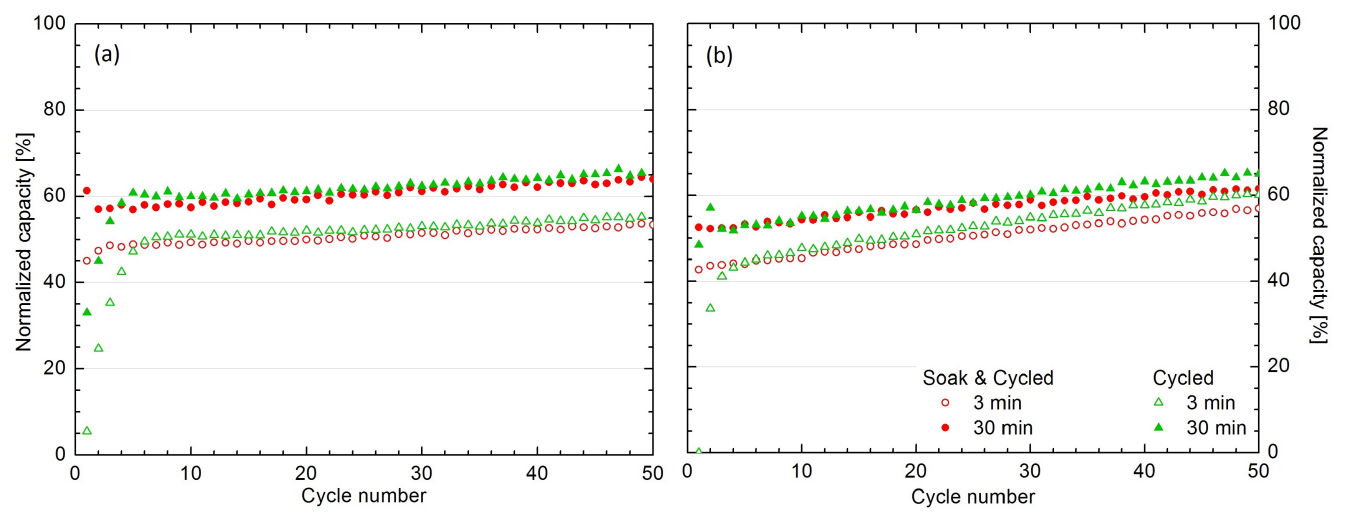

Figure 5: Comparing the normalized hydrogen capacity after 3 and 30 min against cycle number for (a) Mg30 and (b) V_Mg30 when cycled at $400{ }^{\circ} \mathrm{C}$ with/without undergoing a prior soaking process.

minutes, with SS_Mg30 and Fe_Mg30 exceeding the capacity of Mg30 but the opposite occurred for Cr_Mg30 and V_Mg30.

The effect of the coatings on total capacity can be seen more clearly in Figure 6, in which uptake was monitored over a $14 \mathrm{~h}$ period for the $51^{\text {st }}$ cycle. All of the coatings, with the exception of Fe, were found to have a detrimental impact by up to $6.5 \%$ on the final capacity of the $\mathrm{Mg}$ powders. Overall, the

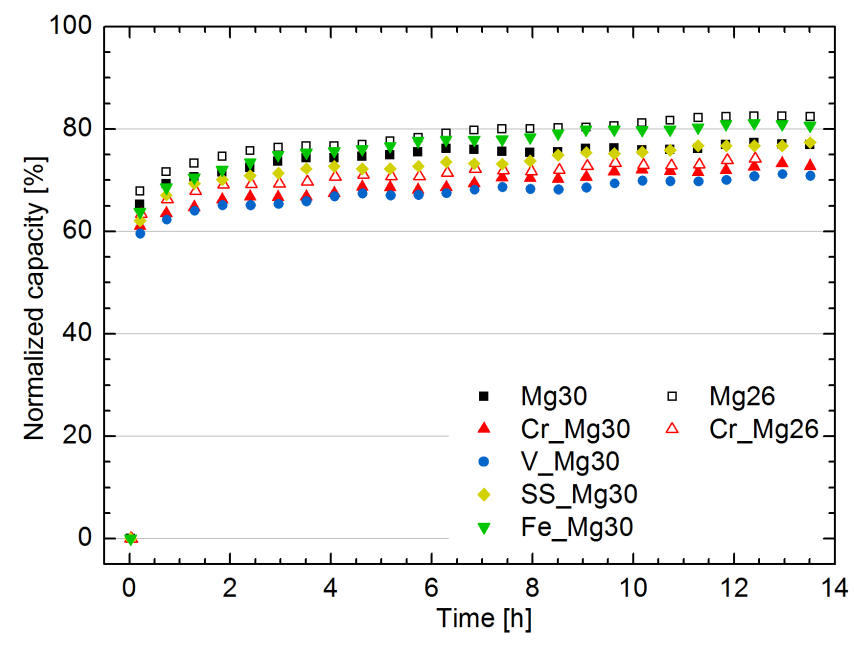

Figure 6: Normalized capacity versus time for each soak \& cycled sample at $400{ }^{\circ} \mathrm{C}$ during an extended final hydrogenation cycle post 50 cycles. 
uncoated Mg26 continued to display the best hydrogenation characteristics achieving a final capacity of $82.5 \%$ after $14 \mathrm{~h}$.

\subsection{Structural Analysis}

Images (a) and (b) in Figure 7 display SEM micrographs of the as received uncoated powders Mg30 and Mg26, validating the high density of spherical particulates present with sizes that agree with the Mastersizer particle size analysis. As expected, after cycling at $350{ }^{\circ} \mathrm{C}$ (images (c) and (d) in Figure 7) both powders underwent almost identical microstructural evolution, with the original spherical particulates still discernible among the sintered clusters that have formed. The surface topology of these surrounding clusters is highly irregular compared to the smooth particulates in images (a) and (b) and is associated with the conjoining of numerous smaller particles during cycling.

Porous structures were also fabricated during the cycling of the coated samples at $350{ }^{\circ} \mathrm{C}$, however substantial morphological differences are evident as presented by the cross-sectional images (e) to (h) in Figure 7. Both V_Mg30 and SS_Mg30 shown in images (e) and (f) respectively, exhibit significant microstructural changes with no noticeable retention of the starting spherical particulates and instead resemble clusters of spheroid globules (ca. $5 \mu \mathrm{m}$ ) sintered together. Image (d) reveals Fe_Mg30 displays distinct similarities to the cycled uncoated powders as some starting spherical particulates remain distinguishable, although there is also more pronounced surface evolution in line with V_Mg30 and SS_Mg30. Cr_Mg26 shown in image (h) experienced the most extensive transformation with signs of more elongated globule (ca. $10 \mu \mathrm{m})$ agglomerations.

Lastly, it is clear that following cycling the uncoated samples possess considerable voids (ca. $50 \mu \mathrm{m}$ ) between the sintered clusters, where as the agglomerations formed among the coated samples are more evenly distributed throughout the overall structures, with the possible exclusion of Fe_Mg30.

\section{Discussion}

The behaviour of Mg30 with successive cycling at $350{ }^{\circ} \mathrm{C}$ (Figure 4(a)), in which over 90 cycles the capacity gradually dropped to slightly below that measured during the $1^{\text {st }}$ cycle, is validated by Garrier et al. [23]. When an intermediate scale tank $\left(1.8 \mathrm{~kg}\right.$ ) of milled $\mathrm{MgH}_{2}$ compacted with 5 wt.\% expanded natural graphite was cycled 50 times at $320^{\circ} \mathrm{C}$, they observed a capacity decrease of about 1 wt.\%, in conjunction with a slight decline in 


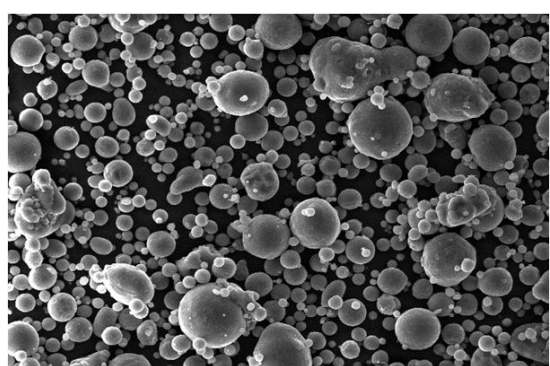

(a)

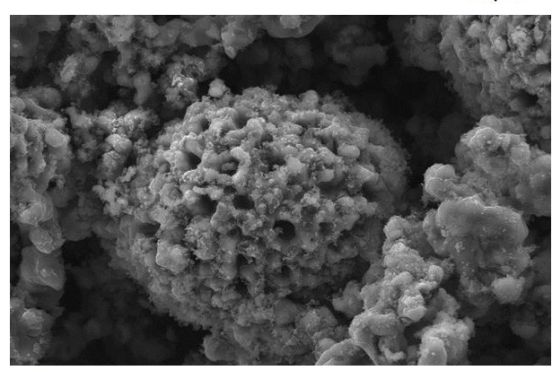

(c)

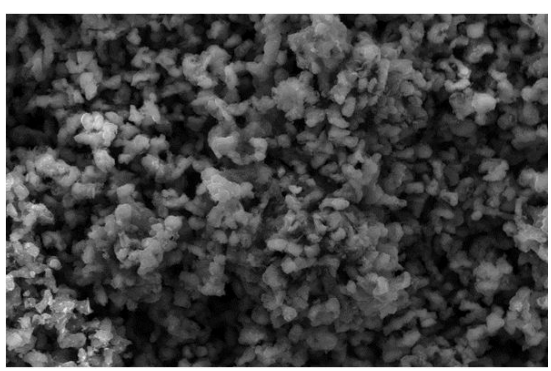

(e)

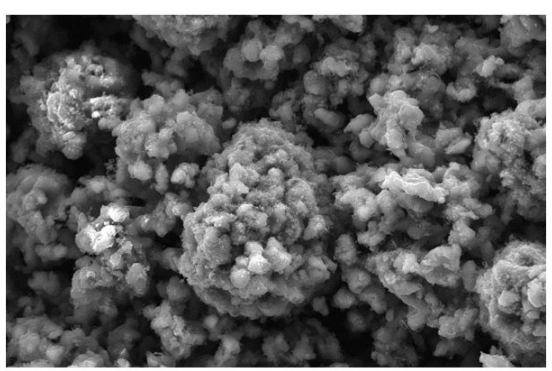

(g) $\overline{20 \mu \mathrm{m}}$

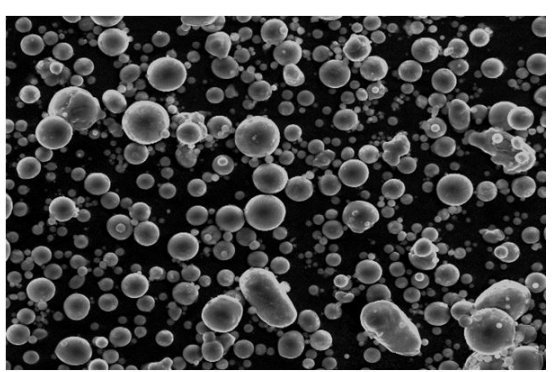

(b)

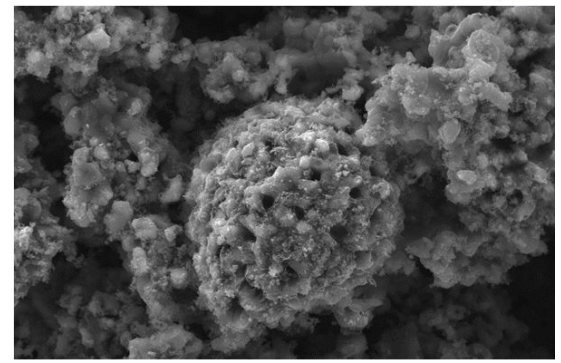

(d)

$\overline{20 \mu \mathrm{m}}$

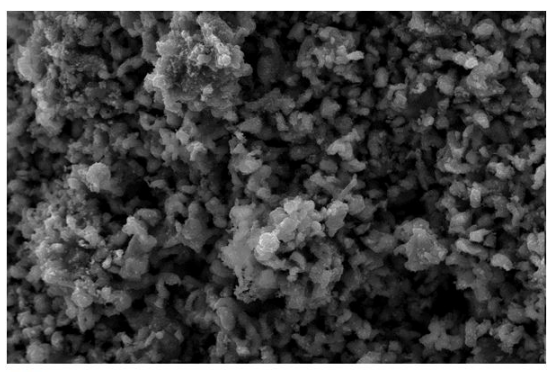

(f)

$\overline{20 \mu \mathrm{m}}$

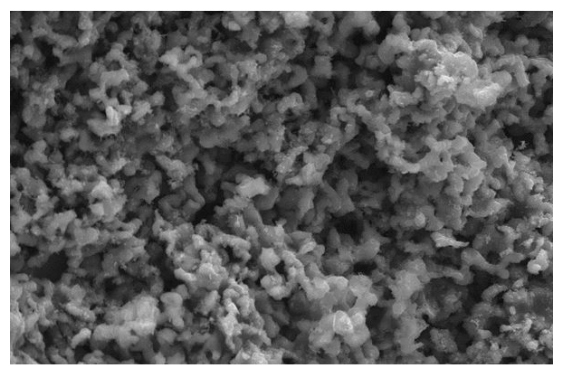

(h)

$\overline{20 \mu \mathrm{m}}$

Figure 7: SEM images of the as received powders (a) Mg30 (AR) and (b) Mg26 (AR). Crosssectional fracture surface SEM images at the midpoints of the porous samples produced following 90 cycles at $350{ }^{\circ} \mathrm{C}$ where (c) Mg30; (d) Mg26; (e) V_Mg30; (f) SS_Mg30; (g) Fe_Mg30 and (h) Cr_Mg26. 
hydrogenation kinetics, during the initial 20 cycles after which the capacity stabilized for the remaining cycles, much like Mg30. Contrastingly, Pedersen et al. [15] reported a consistent drop in capacity, totaling approximately 1 wt. $\%$, after the first 100 cycles at $390{ }^{\circ} \mathrm{C}$. This difference has been attributed to the lower cycling temperature of Mg30 restricting the degree of sintering in the sample and thus also grain recrystallisation. That being said, the capacity of $26.5 \%$ observed for Mg30 in cycle 90 (Figure 3(a)) was drastically lower than the $96 \%$ normalized capacity achieved by $\mathrm{MgH}_{2}$ milled for $20 \mathrm{~h}$ (crystallite size ca. $20 \mathrm{~nm}$ ) that was hydrogenated at $300{ }^{\circ} \mathrm{C}$ [7]. For $\mathrm{Mg} 30$ the absorption kinetics appear to be limited by diffusion through the formed hydride layer and this is verified by the final extended hydrogenation at 400 ${ }^{\circ} \mathrm{C}$ (Figure 6) in which $\mathrm{Mg} 30$ reached a more respectable capacity of $77 \%$ after $14 \mathrm{~h}$.

The behaviour of V_Mg30 with successive cycling at $350{ }^{\circ} \mathrm{C}$ (Figure $4(\mathrm{~b})$ ) indicates not only that material degeneration is prevented with catalyst addition, agreeing with literature, but also the catalysed samples tested here experience a noticeable enhancement from their initial storage properties. Dehouche et al. [20, 21] reported catalyst dependent rises in capacity of $0.2-0.5$ wt. $\%$ and little change in the microstructure or specific surface area of the milled materials were detectable even after 2000 cycles. On the other hand, following only 90 cycles the microstructure of V_Mg30 (Figure 7 (e)) is altered from $30 \mu \mathrm{m}$ spherical particulates (Figure 7 (a)) into a series of sintered spheroid globules (ca. $5 \mu \mathrm{m}$ ), which may have contributed to the capacity increase of $20 \%$.

For hydrogenation at $350{ }^{\circ} \mathrm{C}$ the benefits from the catalyst coatings are clear, not only in terms of initial activation (Figure 1) but also the kinetic and capacity improvements with cycling (Figure 3(b)). Comparing Mg30 and V_Mg30 at $350{ }^{\circ} \mathrm{C}$ (Figure 4) vanadium promotes faster reaction kinetics and increased capacity, which improve with each cycle until plateauing in performance by the $90^{\text {th }}$ cycle. In the $1^{\text {st }}$ cycle V_Mg30 demonstrates a rate of reaction of $19.9 \% \mathrm{~min}^{-1}$ during the initial $30 \%$ of uptake that is indeed faster than the diffusion limited, latter section of the curve $\left(0.2 \% \mathrm{~min}^{-1}\right.$ from 10 min onwards). By the $90^{\text {th }}$ cycle nucleation was now the rate determining step for at least the initial $50 \%$ of uptake in which the reaction rate reached $24.2 \% \mathrm{~min}^{-1}$, possibly indicating the creation of new nucleation sites with cycling. Excluding Fe_Mg30, similar results are observed for the remaining coated samples (Figure S3).

As anticipated, the activation energies (Table 2) determined for uncoated 
Mg30 and Mg26 were found to lie between that of published values for as received $70 \mu \mathrm{m} \mathrm{MgH}_{2}\left(206 \mathrm{~kJ} \mathrm{~mol}^{-1}\right)$ [24] and $20 \mu \mathrm{m} \mathrm{MgH}_{2}\left(156 \mathrm{~kJ} \mathrm{~mol}^{-1}\right)$ [7]. The catalysts reduced the activation energy, however they remain greater than similar thin film structures cycled up to 60 times between $250{ }^{\circ} \mathrm{C}$ and $320{ }^{\circ} \mathrm{C}$, such as $\mathrm{Mg}+3.3$ wt. $\% \mathrm{Cr}\left(65.7 \pm 2.5 \mathrm{~kJ} \mathrm{~mol}^{-1}\right)[25]$ and $\mathrm{Mg}+5.2$ wt.\% V $\left(67.6 \pm 1.2 \mathrm{~kJ} \mathrm{~mol}^{-1}\right)$ [25]. Amirkhiz et al. [19] demonstrated that the reduced activation energies of catalysed as-milled Mg returns back to its initial unmilled value upon successive cycling. For example, they reported the activation energy of milled $\mathrm{Mg}+10 \mathrm{at} . \%$ Fe to increase from $92 \mathrm{~kJ} \mathrm{~mol}{ }^{-1}$ to $119 \mathrm{~kJ} \mathrm{~mol}^{-1}$ following 75 cycles at $250{ }^{\circ} \mathrm{C}$. This post cycling activation energy is more comparable with the values reported for these samples (Table 2) suggesting catalysed atomised Mg powder behaves more like ball milled catalysed Mg than their thin film forms. This was not unforeseen given the homogeneous catalyst distributions achievable in thin film structures compared to their bulk counterparts and it must be noted the catalyst concentrations for the atomised $\mathrm{Mg}$ samples were between 20-50\% less than those in the reported thin film materials.

The fragmentation of the coated spherical magnesium particulates into clusters of spheroid globules (Figure 7 (e), (f) \& (h)) is speculated to generate new nucleation sites and maintain a good catalyst distribution throughout the material resulting in the evolution of kinetics (Figure 3(b)) and the determined activation energies in Table 2. This higher density of nucleation sites means more hydrogen can avoid having to diffuse through layers of hydride to reach new Mg. For example, at around $400{ }^{\circ} \mathrm{C}$ the diffusion coefficient of hydrogen within the hydride phase $\left(2.78 \times 10^{-12} \mathrm{~m}^{2} \mathrm{~s}^{-1}[26]\right)$ is three orders of magnitude lower compared to the unhydrided phase $\left(3 \times 10^{-9}\right.$ $\left.\mathrm{m}^{2} \mathrm{~s}^{-1}[27,28]\right)$, thus the reaction rate during initial stages was substantially improved. Furthermore, as the porous structure of the cycled coated samples consist of voids of $<50 \mu \mathrm{m}$ the critical hydride layer thickness at which further hydrogenation is halted $(30-50 \mu \mathrm{m})[14]$ can be significantly limited. In turn, this may prevent the rate limiting effects of diffusion becoming prominent, and the hydrogenation of more available $\mathrm{Mg}$ can occur unhindered compared to the uncoated samples, as reflected in Figure 3.

The cause of the different microstructural evolution between the coated and uncoated samples during cycling at $350{ }^{\circ} \mathrm{C}$ is speculated to be an extension of a complex process in which particle sintering competes with particle fragmentation (see Figure 8). A degree of structure in the sample can be created by restriction of particle reorientation during successive cycling under 
hydrogen. Given the reasonable self-diffusion coefficient of $\mathrm{Mg}$ atoms at 350 ${ }^{\circ} \mathrm{C}\left(9.26 \times 10^{-16} \mathrm{~m}^{2} \mathrm{~s}^{-1}[29]\right)$, this may occur through the prior formation of sintered bridges at points of contact between particles, which remain in the metallic state for longer, due to slower hydrogenation kinetics as shown. Reinforcement of the bridges occurs upon the onset of dehydrogenation when the surface of the particles transform to the metallic state, yet their cores are still hydrogenated and thus have not undergone particle contraction. Successive cycling causes the process to renew.

For the cycled coated samples at $350^{\circ} \mathrm{C}$, it is postulated particle fragmentation may dictate evolution opposed to particle sintering. Due to their faster kinetics, the formation and growth of the hydride state during hydrogenation may occur too rapidly to enable the generation of sintered bridges at contact points. In addition, sintered bridge formation was not possible at contact points where the catalysts were present given their immiscibility with magnesium at these temperatures and significantly higher melting points. Upon

\section{Uncoated Mg}

(a) Prior to cycling

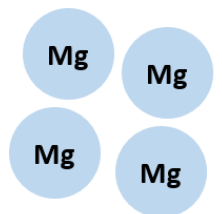

Coated Mg

(a) Prior to cycling

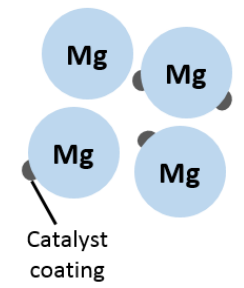

(b) Hydrogenation

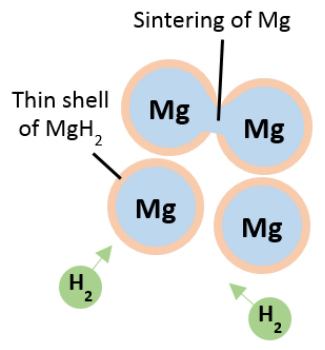

(b) Hydrogenation

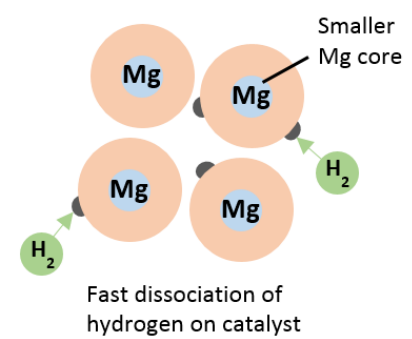

(c) Dehydrogenation

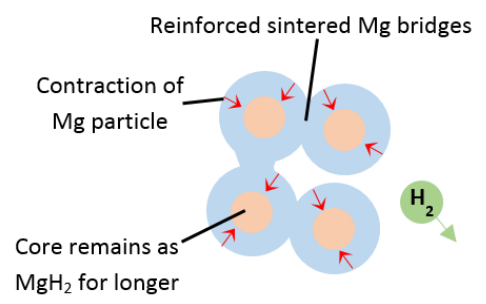

(c) Dehydrogenation

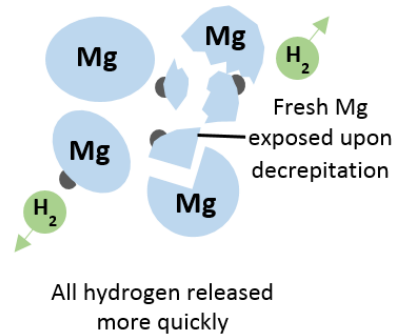

Figure 8: Schematic illustrating the different suggested mechanisms for the evolution of uncoated $\mathrm{Mg}$ and coated $\mathrm{Mg}$ samples with (a) prior to cycling (b) the onset of hydrogenation and (c) during dehydrogenation. 
dehydrogenation, complete transition to the metallic state, along with particle contraction, is too fast preventing sintering. In conjunction, decrepitation of the particles to $5 \mu \mathrm{m}$ spheroid globules enables the catalysts, formerly on the outer surfaces of particles, to diffuse into freshly exposed $\mathrm{Mg}$ surface which is likely to have given rise to the progressive hydrogenation improvements observed with cycling.

The results of Fe_Mg30 help to validate this concept acting as a midpoint between the uncoated and coated samples given its low catalyst concentration. During the activation process at $350{ }^{\circ} \mathrm{C}$ (Figure 1) it was clearly the slowest of the coated samples to reach saturation and it also underwent an incubation period like Mg26 and Mg30. While some benefits of the Fe catalyst were seen during cycling (Figure 3(b)), the kinetic evolution follows a similar trend to Mg30 in that nucleation does not become more prominent upon cycling (Figure S3), with an activation energy and microstructural traits (Figure 7 (g)) that lie midway between that of the uncoated and coated samples. Therefore, for Fe_Mg30 a steady state point may have been reached between particle sintering and particle fragmentation.

Successive cycling at $400{ }^{\circ} \mathrm{C}$ was found to provide no noteworthy benefit to the hydrogenation characteristics of $30 \mu \mathrm{m}$ and $26 \mu \mathrm{m}$ commercial grade atomised magnesium as indicated by the results in Figures 3(b), 5 and 6 . Besides minor improvements in the hydrogenation kinetics of the samples, the coatings have an overall detrimental impact on the final capacity, as highlighted in Figure 6. These findings are in agreement with Huhn et al. [30] who reported a drastic reduction in kinetics and capacity of ball milled $\mathrm{MgH}_{2}$ with $0.5 \mathrm{~mol} \% \mathrm{Nb}_{2} \mathrm{O}_{5}$ measured at $300{ }^{\circ} \mathrm{C}$ after annealing for $5 \mathrm{~h}$ at temperatures greater than $370{ }^{\circ} \mathrm{C}$. This suggests regardless of the processing technique, type of additive and experimental conditions utilised, if catalystdoped magnesium is exposed to temperatures exceeding $370{ }^{\circ} \mathrm{C}$ for extended periods $(>5 \mathrm{~h}$ ) there is a significant deterioration in hydrogenation properties.

Huhn et al. [30] proposed since $\mathrm{MgO}$ is more stable than $\mathrm{Nb}_{2} \mathrm{O}_{5}$, at these temperatures $\mathrm{Mg}$ reduces $\mathrm{Nb}_{2} \mathrm{O}_{5}$ forming $\mathrm{MgO}$ which impedes hydrogen transport. $\mathrm{MgO}$ is also more thermodynamically stable than the metal oxide states of the catalysts used here, although no distinct $\mathrm{MgO}$ peak was identified in the XRD analysis of the cycled samples (e.g. V_Mg30, Figure S4). This was not unexpected given the low catalyst concentrations used in these experiments and suggests poisoning of $\mathrm{Mg}$ causing a capacity reduction may be a smaller effect. Instead the primary cause may relate to the lack of multivalent oxide state of the catalysts, brought about by reduction of the 
catalysts to their metallic states. Research has indicated transition metal oxides act as better catalysts than their metal counterparts [31, 32, 33], yet metal catalysts have still been reported to enhance reaction kinetics of $\mathrm{MgH}_{2}$ $[18,34]$. Therefore, even if the catalysts were reduced completely to their metallic states, improved storage properties compared to Mg26 and Mg30 would still be anticipated. However, as this was not the case, this lends support to the idea that the kinetic benefits is dependent on the multivalency of the transition metal in the oxide compound [33].

The effect of different activation mechanisms were also examined at 400 ${ }^{\circ} \mathrm{C}$. Samples which underwent activation by cycling required 5 cycles before plateauing at the same hydrogenation kinetics and total capacity as the samples which were activated by soaking prior to cycling (Figure 5). The duration for 5 cycles is $5 \mathrm{~h}$ and this is approximately equivalent to the saturation time for the soak and cycled samples during the soaking process (Figure S5). This supports theory that activation of $\mathrm{Mg}$ is owed to the small concentration of highly catalytically active oxide vacancies formed during heating to $>300{ }^{\circ} \mathrm{C}[35,36]$ rather than the breakdown of the inactive $\mathrm{MgO}$ surface layer during successive expansion/contraction cycles.

\section{Conclusion}

Elevated temperature cycling studies were performed on commercial gas atomised $\mathrm{Mg}$ powder with magnetron sputtered catalysts applied to their surfaces. At $350{ }^{\circ} \mathrm{C}$, the benefit of catalyst coatings on the hydrogen storage properties of atomised $\mathrm{Mg}$ powders are evident during activation and successive cycling up to 90 times. The material undergoes different microstructural evolution during cycling when in the presence of a surface catalyst, causing an enhancement of the fast nucleation stage of (de)hydrogenation. This was attributed to particle fragmentation dominating particle sintering, whereas the opposite occurs for the uncoated material. Combining these findings it is proposed that this increased density of nucleation sites causes a sufficient number of particles to transform into the hydride state before adequate generation of sintered particle bridges is possible, which would otherwise act to restrict particle fragmentation. In turn, greater decrepitation of the coated powders allows the diffusion of the catalyst into fresh exposed $\mathrm{Mg}$ surfaces causing the progressive hydrogenation improvements observed.

These results demonstrate under certain conditions magnetron sputtered catalysts do provide a route to using gas atomised $\mathrm{Mg}$ powder as thermal 
storage media for concentrated solar power applications, as they provide a mechanism by which the detrimental effects that spherical particles have on activation and kinetics are overcome.

\section{Acknowledgements}

We would like to acknowledge Dr D. Ievlev and Dr K. Cooke for the coating work carried out by Teer Coatings Ltd, Miba Coating Group and Innovate UK (formerly Technology Strategy Board) for providing funding for this work to be undertaken as part of the HStore project.

\section{References}

[1] IEA, Technology Roadmaps for Solar Electricity (2014) 1-18.

[2] DOE, SunShot - Concentrating Solar Power Program Review.

[3] D. Harries, M. Paskevicius, D. Sheppard, T. Price, C. Buckley, Concentrating Solar Thermal Heat Storage Using Metal Hydrides, Proceedings of the IEEE 100 (2) (2012) 539-549.

[4] S. Kuravi, J. Trahan, D. Goswami, M. Rahman, E. Stefanakos, Thermal energy storage technologies and systems for concentrating solar power plants, Progress in Energy and Combustion Science 39 (4) (2013) 285319.

[5] A. Zaluska, L. Zaluski, J. Ström-Olsen, Nanocrystalline magnesium for hydrogen storage, Journal of Alloys and Compounds 288 (1-2) (1999) $217-225$.

[6] A. Zaluska, L. Zaluski, J. Ström-Olsen, Structure, catalysis and atomic reactions on the nano-scale: a systematic approach to metal hydrides for hydrogen storage, Applied Physics A 72 (2) (2001) 157-165.

[7] J. Huot, G. Liang, S. Boily, A. Van-Neste, R. Schulz, Structural study and hydrogen sorption kinetics of ball-milled magnesium hydride, Journal of Alloys and Compounds 293-295 (1999) 495-500. 
[8] N. Hanada, T. Ichikawa, S. Orimo, H. Fujii, Correlation between storage properties and structural characteristics in mechanically milled magnesium hydride $\mathrm{MgH}_{2}$, Journal of Alloys and Compounds 366 (2004) 269-273.

[9] V. Bérubé, G. Radtke, M. Dresselhaus, G. Chen, Size effects on the hydrogen storage properties of nanostructured metal hydrides: A review, International Journal of Energy Research 31 (6-7) (2007) 636-663.

[10] N. Eigen, C. Keller, M. Dornheim, T. Klassen, R. Bormann, Industrial production of light metal hydrides for hydrogen storage, Scripta Materialia 56 (10) (2007) 847-851.

[11] I. Anderson, R. Terpstra, Progress toward gas atomization processing with increased uniformity and control, Material Science and Engineering A 326 (2002) 101-109.

[12] B. Vigeholm, J. Kjøller, B. Larsen, A. Pedersen, Formation and decomposition of magnesium hydride, Journal of the Less Common Metals 89 (1983) 135-144.

[13] A. Pedersen, J. Kjøller, B. Larsen, B. Vigeholm, Magnesium for hydrogen storage, International Journal of Hydrogen Energy 8 (3) (1983) 205-211.

[14] B. Vigeholm, K. Jensen, B. Larsen, A. Pedersen, Elements of hydride formation mechanisms in nearly spherical magnesium powder particles, Journal of the Less Common Metals 131 (1-2) (1987) 133-141.

[15] A. Pedersen, J. Kjøller, B. Larsen, B. Vigeholm, J. Jensen, Long-term cycling of the magnesium hydrogen system, International Journal of Hydrogen Energy 9 (9) (1984) 799-802.

[16] M. Verga, F. Armanasco, C. Guardamagna, C. Valli, A. Bianchin, F. Agresti, S. Russo, A. Maddalena, G. Principi, Scaling up of Mg hydride in a temperature and pressure-controlled hydrogen storage device, International Journal of Hydrogen Energy 34 (10) (2009) 4602-4610.

[17] W. Oelerich, T. Klassen, R. Bormann, Comparison of the catalytic effects of $\mathrm{V}, \mathrm{V}_{2} \mathrm{O}_{5}, \mathrm{VN}$ and $\mathrm{VC}$ on the hydrogen sorption of nanocrystalline $\mathrm{Mg}$, Journal of Alloys and Compounds 322 (1-2) (2001) L5-L9. 
[18] J. Bystrzycki, T. Czujko, R. Varin, Processing by controlled mechanical milling of nanocomposite powders $\mathrm{Mg}+\mathrm{X}(\mathrm{X}=\mathrm{Co}, \mathrm{Cr}, \mathrm{Mo}, \mathrm{V}, \mathrm{Y}, \mathrm{Zr})$ and their hydrogenation properties, Journal of Alloys and Compounds 404-406 (2005) 507-510.

[19] B. Amirkhiz, B. Zahiri, P. Kalisvaart, D. Mitlin, Synergy of elemental Fe and Ti promoting low temperature hydrogen sorption cycling of magnesium, International Journal of Hydrogen Energy 36 (11) (2011) $6711-6722$.

[20] Z. Dehouche, R. Djaozandry, J. Huot, S. Boily, J. Goyette, T. Bose, R. Schulz, Influence of cycling on the thermodynamic and structure properties of nanocrystalline magnesium based hydride, Journal of Alloys and Compounds 305 (1-2) (2000) 264-271.

[21] Z. Dehouche, T. Klassen, W. Oelerich, J. Goyette, T. Bose, R. Schulz, Cycling and thermal stability of nanostructured $\mathrm{MgH}_{2}-\mathrm{Cr}_{2} \mathrm{O}_{3}$ composite for hydrogen storage, Journal of Alloys and Compounds 347 (1-2) (2002) 319-323.

[22] S. Sabitu, A. Goudy, Dehydrogenation Kinetics and Modeling Studies of $\mathrm{MgH}_{2}$ Enhanced by Transition Metal Oxide Catalysts Using Constant Pressure Thermodynamic Driving Forces, Metals 2 (3) (2012) 219-228.

[23] S. Garrier, A. Chaise, P. de Rango, P. Marty, B. Delhomme, D. Fruchart, S. Miraglia, $\mathrm{MgH}_{2}$ intermediate scale tank tests under various experimental conditions, International Journal of Hydrogen Energy 36 (16) (2011) 9719-9726.

[24] A. Patah, A. Takasaki, J. Szmyd, Influence of multiple oxide $\left(\mathrm{Cr}_{2} \mathrm{O}_{3} / \mathrm{Nb}_{2} \mathrm{O}_{5}\right)$ addition on the sorption kinetics of $\mathrm{MgH}_{2}$, International Journal of Hydrogen Energy 34 (7) (2009) 3032-3037.

[25] C. Fry, D. Grant, G. Walker, Catalysis and evolution on cycling of nano-structured magnesium multilayer thin films, International Journal of Hydrogen Energy 39 (2) (2014) 1173-1184.

[26] J. Čermák, L. Král, Hydrogen diffusion in Mg-H and Mg-Ni-H alloys, Acta Materialia 56 (12) (2008) 2677-2686. 
[27] J. Renner, H. Grabke, Determination of diffusion coefficients in the hydriding of alloys, Journal of Physical Metallurgy 69 (10) (1978) 639642 .

[28] M. Ramzan, T. Hussain, R. Ahuja, Hydrogen diffusion in bulk and nanoclusters of $\mathrm{MgH}_{2}$ and the role of catalysts on the basis of ab initio molecular dynamics, Applied Physics Letters 94 (22) (2009) 221910.

[29] N. Kulkarni, R. Warmack, B. Radhakrishnan, J. Hunter, Y. Sohn, K. Coffey, G. Murch, I. Belova, Overview of SIMS-Based Experimental Studies of Tracer Diffusion in Solids and Application to Mg Self-Diffusion, Journal of Phase Equilibria and Diffusion 35 (6) (2014) 762-778.

[30] P. Huhn, M. Dornheim, T. Klassen, R. Bormann, Thermal stability of nanocrystalline magnesium for hydrogen storage, Journal of Alloys and Compounds 404-406 (2005) 499-502.

[31] G. Barkhordarian, T. Klassen, R. Bormann, Fast hydrogen sorption kinetics of nanocrystalline $\mathrm{Mg}$ using $\mathrm{Nb}_{2} \mathrm{O}_{5}$ as catalyst, Scripta Materialia 49 (3) (2003) 213-217.

[32] D. Croston, D. Grant, G. Walker, The catalytic effect of titanium oxide based additives on the dehydrogenation and hydrogenation of milled $\mathrm{MgH}_{2}$, Journal of Alloys and Compounds 492 (1-2) (2010) 251-258.

[33] W. Oelerich, T. Klassen, R. Bormann, Metal oxides as catalysts for improved hydrogen sorption in nanocrystalline Mg-based materials, Journal of Alloys and Compounds 315 (1-2) (2001) 237-242.

[34] J. Bobet, E. Akiba, Y. Nakamura, B. Darriet, Study of Mg-M (M = $\mathrm{Co}, \mathrm{Ni}$ and $\mathrm{Fe}$ ) mixture elaborated by reactive mechanical alloying hydrogen sorption properties, International Journal of Hydrogen Energy 25 (10) (2000b) 987-996.

[35] Y. Gu, A. Brenner, Hydrogen Activation by Magnesia Catalysts, Journal of Catalysis 136 (1) (1992) 222-231.

[36] A. Borgschulte, M. Bielmann, A. Züttel, G. Barkhordarian, M. Dornheim, R. Bormann, Hydrogen dissocation on oxide covered $\mathrm{MgH}_{2}$ by catalytically active vacancies, Applied Surface Science 254 (8) (2008) $2377-2384$. 